\title{
A proton NMR study of the effect of a new intravasal injectable male contraceptive RISUG on seminal plasma metabolites
}

\author{
U. Sharma ${ }^{1}$, K. Chaudhury ${ }^{2}$, N. R. Jagannathan ${ }^{1}$ and S. K. Guha ${ }^{3 *}$ \\ ${ }^{1}$ Department of NMR, All India Institute of Medical Sciences, New Delhi 110 029, India; \\ ${ }^{2}$ Centre for Biomedical Engineering, Indian Institute of Technology, New Delhi 110016 , \\ India; and ${ }^{3}$ Centre for Biomedical Engineering, Indian Institute of Technology and \\ All India Institute of Medical Sciences, New Delhi 110 029, India
}

\begin{abstract}
Nuclear magnetic resonance (NMR) spectroscopy was used to quantify citrate, glucose, lactate, glycerophosphorylcholine and choline in seminal plasma from subjects injected with a new male contraceptive RISUG, a copolymer of styrene maleic anhydride dissolved in dimethyl sulphoxide, and in seminal plasma from normal ejaculates. No significant difference in the concentration of citrate was observed between the groups, indicating that the prostate is not affected by the contraceptive. The concentrations of glucose, lactate, glycerophosphorylcholine and choline were significantly lower $(P<0.01)$ in
\end{abstract}

subjects injected with RISUG compared with controls. In addition, metabolite ratios such as choline:citrate, citrate:lactate, choline:lactate and glycerophosphorylcholine:choline were calculated. Citrate:lactate and glycerophosphorylcholine:choline ratios were significantly lower in RISUG-injected subjects than in controls $(P<0.01)$, thereby indicating the occurrence of partial obstructive azoospermia. The most important finding of the present study was that the intervention of RISUG in the vas deferens even for a period as long as 8 years is absolutely safe and does not lead to prostatic diseases.

\section{Introduction}

There is a need to develop a suitable male contraceptive for population control programmes. The current unavailability of an ideal male contraceptive is due to the lack of one or more essential features such as a minimally invasive drug delivery system with a one-time intervention, long-term effectiveness of the contraceptive with negligible sideeffects and the option of reversal whenever desired. Rigorous research has been carried out over the past 25 years to meet these objectives, which has led to the successful development of a reversible male contraceptive that can be injected bilaterally into the lumen of the vas deferens. The contraceptive, named RISUG, consists of a specific preparation of the copolymer styrene maleic anhydride dissolved in dimethyl sulphoxide (DMSO). When spermatozoa come into contact with RISUG in the vas deferens, their membranes rupture and enzymes such as acrosin and hyaluronidase leach out (Guha, 1996). Hence, these damaged spermatozoa are rendered incapable of fertilization. Phase I and phase II clinical trials have provided guidelines for drug dose selection (Guha et al., 1993) and established the effectiveness of a specific dose of the contraceptive (Guha et al., 1997). A phase III clinical trial is currently underway. Toxicological studies (Sethi et

*Correspondence

Email: guhask@cbme.iitd.ernet.in al., 1990), including teratogenic safety on reversal (Sethi et al., 1992), have also been conducted. RISUG is non-toxic and safe, and has long-term effectiveness. The compound is retained in the vas deferens even though it is non-sclerosing and not tissue adherent. In addition, reversibility can be achieved easily in a minimally invasive manner (Guha, 1999).

Human seminal plasma contains a mixture of fluid from the seminiferous tubules, epididymis and accessory glands (bulbo-urethral, prostate and seminal vesicles). The composition of each secretion has characteristic biochemical features: fructose and prostaglandins are markers for seminal vesicles; prostatic fluid contains uniquely high concentrations of citrate (Costello and Franklin, 1991), zinc, polyamines and myo-inositol; and glycerophosphorylcholine, choline, neutral $\alpha$-glucosidase and carnitine are markers of epididymal function (Cooper et al., 1990). High-resolution ${ }^{1} \mathrm{H}$ NMR spectroscopy has emerged as a powerful tool in the analysis of biofluids (Foxall et al., 1993; Spraul et al., 1994; Liu et al., 1996). Recent studies have shown that chemometric methods along with automated NMR-based analysis can be used for toxicological assessment of biofluids (Holmes et al., 2000). Lynch et al. (1994) showed that NMR could be used successfully as a diagnostic probe for altered male reproductive gland function. ${ }^{1} \mathrm{H}$ NMR spectroscopy has also been used to examine seminal plasma of patients in an IVF programme (Segalen et al., 1995). Recently, Hamamah et al. (1998) showed that seminal plasma metabolite ratios could be used to 
differentiate between cases of spermatogenic failure and obstructive azoospermia. The main advantage that NMR spectroscopy offers over other classical biochemical analyses is that it is not biased towards a particular compound; it can simultaneously detect metabolites that are expected or unexpected or that are difficult to assay using standard biochemical methods (Patel et al., 1999). There is also no need for specific preparation such as derivatization of compounds.

The aim of the present study was to evaluate prostatic function in RISUG-injected subjects by determining the concentration of citrate in the seminal plasma ejaculates. In addition, glucose, lactate, glycerophosphorylcholine and choline metabolites were quantified. Peak area ratios of these metabolites were also estimated to assess the occurrence of obstructive azoospermia in RISUG-injected subjects.

\section{Materials and Methods}

\section{Seminal plasma preparation}

Human semen samples were collected by masturbation from (i) 20 normal human volunteers (age 32-40 years; controls) who had fathered children within the previous 4 years and (ii) 17 subjects (aged 36-40 years) who had fathered children before the injection of RISUG given to them 8 years earlier. Each sample was liquefied at $37^{\circ} \mathrm{C}$ for $20 \mathrm{~min}$ to reduce viscosity. A $0.5 \mathrm{ml}$ aliquot was removed to determine semen parameters using standard methods (WHO Recommended Procedure, 1999). The remaining semen sample was centrifuged at $1000 \mathrm{~g}$ for $15 \mathrm{~min}$ to remove cells and spermatozoa. The resulting supernatant was separated, diluted with $\mathrm{D}_{2} \mathrm{O}$ and NMR experiments were performed immediately, within 1 min. Even after separation from spermatozoa by centrifugation, the composition of seminal plasma undergoes progressive changes in vitro, for example, proteolysis, increase in free choline content due to dephosphorylation of phosphorylcholine, and fructolysis. Therefore, the plasma was subjected to NMR analysis immediately after separation of spermatozoa and cells. Identical conditions were maintained strictly for each sample evaluation.

\section{NMR analysis}

Seminal plasma samples were transferred into $5 \mathrm{~mm}$ NMR sample tubes with $\mathrm{D}_{2} \mathrm{O}$ (Aldrich Company Inc., Milwaukee, IL) for field/frequency lock. Sodium 3(trimethylsilyl-2,2,3,3-H)-1-propionate (TSP) (E-Merck, Darmstadt) was added at a concentration of $2 \mathrm{mmol} \mathrm{I}^{-1}$ to the seminal plasma samples to serve as a chemical shift and concentration reference.

The spectra were recorded on a Bruker DRX-400 spectrometer $(9.4 \mathrm{~T})$ operating at $400.13 \mathrm{MHz}$ for protons at $25^{\circ} \mathrm{C}$. Typical parameters for one-dimensional NMR experiments were: pulse width $90^{\circ}$, spectral width $5 \mathrm{kHz}$ and $32-64$ scans. A long relaxation delay of $14 \mathrm{~s}$ was used for quantitative measurements. A constant receiver gain was used for all one-dimensional experiments to minimize errors in quantitative estimation. The free induction decays (FIDs) were collected with $32 \mathrm{~K}$ data points and line broadening of $0.3 \mathrm{~Hz}$ was applied before Fourier transformation.

Connectivity between protons was established with phase-sensitive double quantum-filtered correlation spectra using a standard pulse sequence. Typically, the following experimental conditions were used: spectral width $5 \mathrm{kHz}$, $2 \mathrm{~K}$ data points in $\mathrm{F} 2$ dimension with $512 \mathrm{t}_{1}$ increments in F1 dimension. The number of scans was 32-64, the relaxation delay was $2.5 \mathrm{~s}$ and the receiver gain was optimized in each instance to obtain good signal:noise ratio. Data were zero filled to $1 \mathrm{~K}$ in F1 dimension. Squared sine bell window function was applied before Fourier transformation.

\section{Quantification of metabolites}

Peaks or multiplets of identified metabolites, including that of internal standard TSP, were integrated to obtain signal intensity after careful baseline correction. The following formula was used to estimate the concentrations of various metabolites:

$$
[C]_{\mathrm{X}}=[C]_{\mathrm{TSP}} \frac{N_{\mathrm{TSP}} \cdot I_{\mathrm{X}}}{N_{\mathrm{X}} \cdot I_{\mathrm{TSP}}}
$$

$[C]_{X}$ is the concentration of biochemical $X$, and $I_{X}$ and $I_{\text {TSP }}$ are the NMR signal intensities of $X$ and TSP, respectively. $N_{X}$ is the number of protons per molecule giving rise to the integrated signal and $N_{\text {TSP }}=9$. Metabolite ratios were calculated from the integrated intensity of individual peaks.

\section{Statistical analysis}

Concentrations of citrate, glucose, lactate, glycerophosphorylcholine and choline are expressed as mean \pm SD. Concentration and peak area ratios of specific biochemical markers from both the groups were compared using Student's $t$ test. The level of significance was set at $P<0.01$.

\section{Results}

Various amino acids, carbohydrates and lipids present in human seminal plasma were assigned in RISUG-injected subjects using $400 \mathrm{MHz}{ }^{1} \mathrm{H}$ NMR spectroscopy. Expanded regions of a typical one-dimensional proton NMR spectrum of seminal plasma of controls and of subjects injected with RISUG are shown (Fig. 1a,b). Several intense resonances were observed in the aliphatic range (0.5-4.5 p.p.m.) and aromatic region (6.5-8.5 p.p.m.). A two-dimensional double quantum filter correlation spectrum of the corresponding regions is shown (Fig. 2). Assignment of the resonances was made using the J-connectivity pattern. Resonances corresponding to $\mathrm{CH}$ and $\mathrm{CH}_{3}$ protons of lactate were 
Table 1. Concentrations of metabolites from the seminal plasma of normal controls and men treated with the intravasal injectable contraceptive RISUG

\begin{tabular}{|c|c|c|c|c|c|c|c|}
\hline \multirow[b]{2}{*}{ Subjects } & \multirow{2}{*}{$\begin{array}{c}\text { Sperm } \\
\text { concentration } \\
\left(\times 10^{-6} \mathrm{ml}^{-1}\right)\end{array}$} & \multirow{2}{*}{$\begin{array}{l}\text { Volume of } \\
\text { ejaculate }\end{array}$} & \multicolumn{5}{|c|}{ Concentration of metabolites $\left(\mathrm{mmol}^{-1}\right)$} \\
\hline & & & Citrate & Glucose & Lactate & GPC & Choline \\
\hline Normal controls $(n=20)$ & $75 \pm 10$ & $4.0 \pm 0.6$ & $55.8 \pm 11.0$ & $7.6 \pm 0.4$ & $15.5 \pm 2.1$ & $4.8 \pm 0.4$ & $7.6 \pm 0.4$ \\
\hline RISUG-injected $(n=17)$ & $<1 \pm 0.2$ & $3.5 \pm 0.5$ & $54.4 \pm 9.0$ & $3.6 \pm 0.2^{*}$ & $8.8 \pm 0.8^{*}$ & $1.0 \pm 0.3^{*}$ & $6.8 \pm 2.2^{*}$ \\
\hline
\end{tabular}

GPC: glycerophosphorylcholine.

Values are mean \pm SD.

*Value is significantly different from corresponding control value $(P<0.01)$.

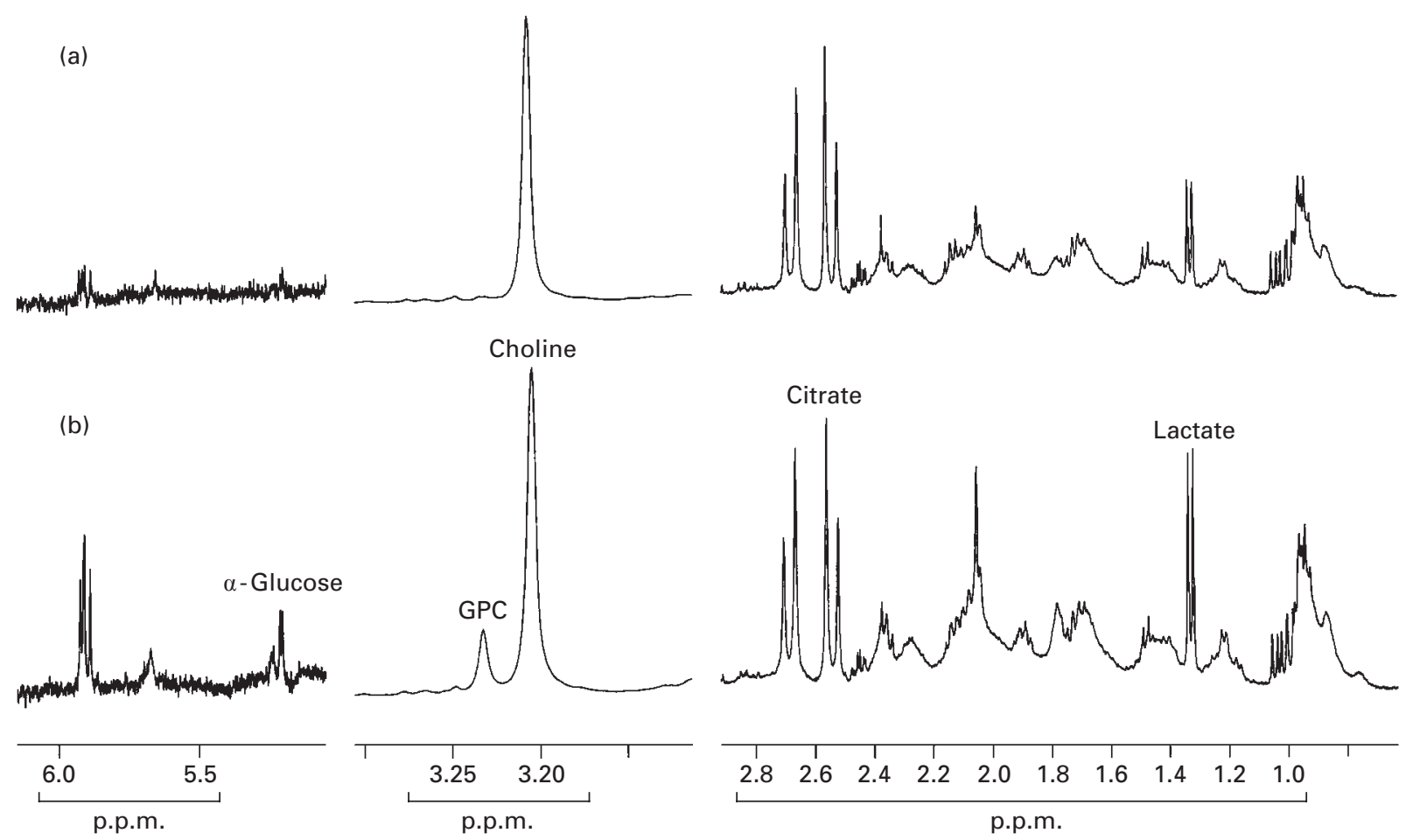

Fig. 1. The expanded regions of one pulse $400 \mathrm{MHz}{ }^{1} \mathrm{H}$ nuclear magnetic resonance spectra of human seminal plasma diluted in $\mathrm{D}_{2} \mathrm{O}$ of (a) subjects injected with the contraceptive RISUG into the lumen of the vas deferens and (b) normal controls. Expanded regions are shown: 0.6-2.9 p.p.m. showing lactate and citrate resonances, scale of signal intensity is magnified by four times; the region 3.10-3.30 p.p.m. shows glycerophosphorylcholine (GPC) and choline resonances, scale is unchanged; and the region 5.0-6.1 p.p.m. shows the resonance of $\alpha$-glucose resonance with scale magnified by eight times

assigned at 4.11 and 1.33 p.p.m., respectively, from the cross peak observed between them in the two-dimensional double quantum filter spectrum. Similarly, the signals from citrate, glucose, glycerophosphorylcholine, myo-inositol and spermine were assigned using the connectivities. No significant difference was observed in the chemical shift positions of metabolites present in the seminal plasma samples of control and RISUG-injected subjects (Fig. 1).

The calculated concentrations of specific biochemical markers of prostate, seminal vesicles and epididymis for control and RISUG-injected subjects are shown (Table 1). Most of these metabolites had reasonably distinct resonance peaks in the one-dimensional spectrum, thereby facilitating reliable estimation of their concentration. The concentration of citrate was estimated from its two doublets at 2.55 and 2.67 p.p.m. No significant difference in the concentration of citrate in the seminal plasma ejaculates was observed between control and RISUG-injected subjects. A distinct resonance corresponding to $\mathrm{H} 1$ ' proton of glucose was observed, which aided determination of glucose concentration. A significant decrease $(P<0.01)$ was observed in the concentrations of glucose, lactate, glycerophosphorylcholine and choline in the seminal plasma ejaculates of RISUG-injected men compared with controls. 


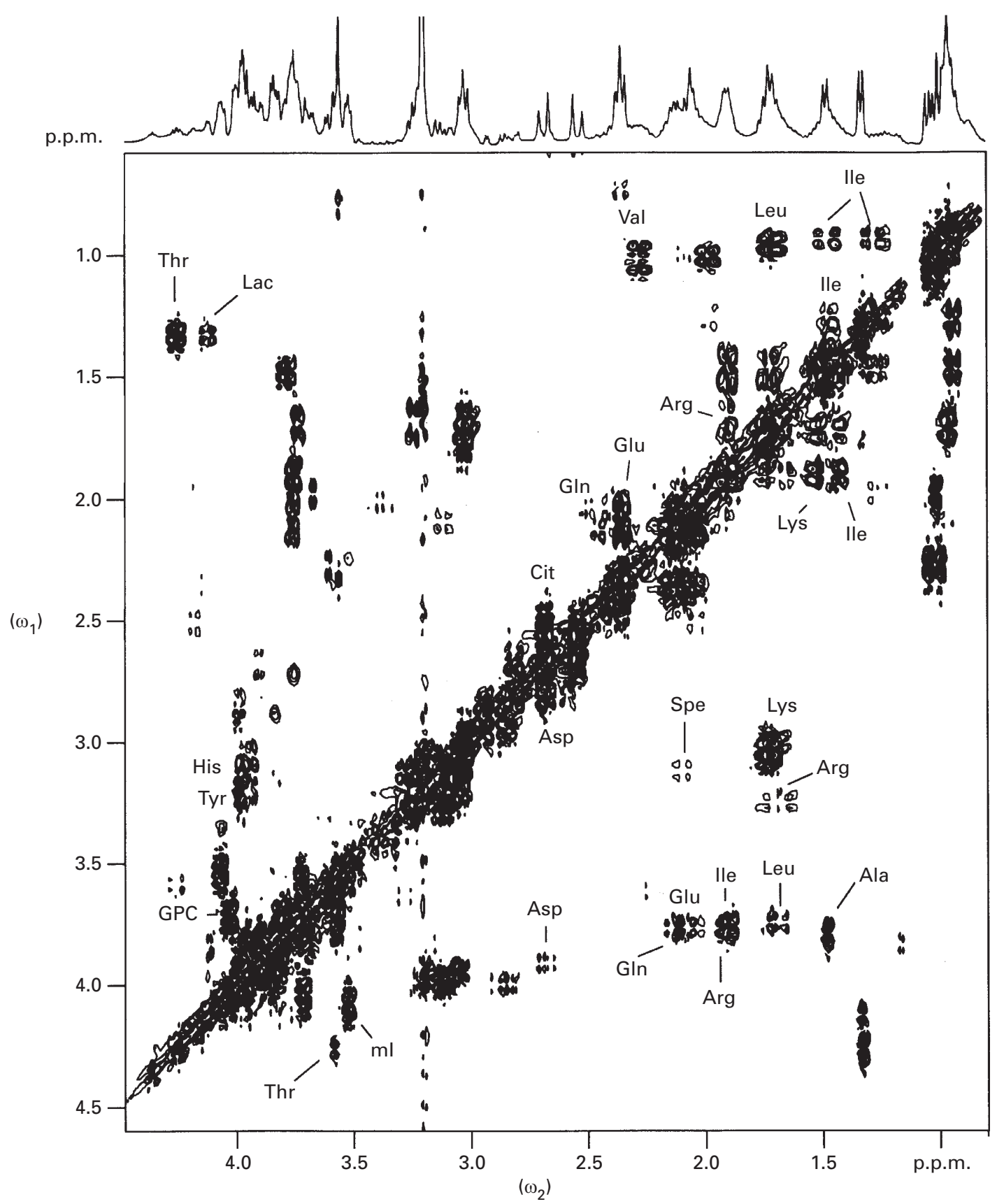

Fig. 2. The expanded aliphatic region of $400 \mathrm{MHz}$ two-dimensional double quantum filtered correlation spectra showing cross peaks connected with J-coupling of human seminal plasma for subjects injected with the contraceptive RISUG into the lumen of the vas deferens. GPC: glycerophosphorylcholine; Thr: threonine; Val: valine; Lac: lactate; Cit: citrate; Leu: leucine; Ile: isoleucine; Arg: arginine; Glu: glutamic acid; Gln: glutamine; Lys: lysine; Spe: spermine; Asp: aspartic acid; His: histidine; Tyr: tyrosine; ml: myo-inositol; and Ala: alanine.

Citrate:lactate and glycerophosphorylcholine:choline ratios were also lower in RISUG-injected subjects $(P<0.01)$ compared with controls (Table 2).

\section{Discussion}

The concentrations of prostatic markers vary in different prostatic diseases. Citrate concentrations are reported to be lowered significantly in prostatic carcinoma (Cornel et al., 1993). Vasectomy, the most commonly used method of male contraception, is suspected to be linked with subsequent prostate cancer (Comhaire, 1994). Therefore, it was considered essential to evaluate the prostatic function in RISUG-injected subjects. In the present study, the prostate biochemical marker citrate, which can be assigned specifically from NMR spectra, was quantified. No 
Table 2. Mean values of peak area ratios of metabolites present in seminal plasma of normal controls and men treated with the intravasal injectable contraceptive RISUG assessed using ${ }^{1} \mathrm{H}$ nuclear magnetic resonance (NMR) spectroscopy

\begin{tabular}{lcc}
\hline Metabolites & $\begin{array}{c}\text { Normal controls } \\
(n=20)\end{array}$ & $\begin{array}{c}\text { RISUG-injected } \\
\text { subjects }(n=17)\end{array}$ \\
\hline Choline:citrate & $4.4 \pm 2.1$ & $2.8 \pm 1.7$ \\
Choline:lactate & $5.9 \pm 2.5$ & $6.0 \pm 2.6$ \\
Citrate:lactate & $1.4 \pm 0.5$ & $2.4 \pm 0.9^{*}$ \\
GPC:choline & $0.14 \pm 0.05$ & $0.08 \pm 0.05^{*}$ \\
\hline
\end{tabular}

GPC: glycerophosphorylcholine.

Values are mean \pm SD.

*Value is significantly different from corresponding control value $(P<0.01)$.

significant difference was observed in the concentration of citrate in control and RISUG-injected men. This finding is of great importance as it implies that intervention of RISUG in the vas deferens, even for a period as long as 8 years, is absolutely safe and does not lead to prostatic diseases. The glucose content determined in normal ejaculates in the present study was in agreement with the biochemical assay results reported by Diamandis et al. (1999). A significant decrease in the glucose content in seminal plasma of RISUG-injected men was observed: the feedback mechanism for decreased synthesis of glucose in seminal vesicles of RISUG-injected subjects cannot be ruled out. The estimated concentration of lactate in normal ejaculates in the present study was slightly higher than that reported after biochemical analysis by Mann and Mann (1981); this finding may be the result of the overlapping of the methyl resonance peak of threonine with the methyl protons of lactate. The decrease in the amount of lactate in the seminal plasma of RISUG-injected men may be attributed to insufficient secretion of the enzyme lactate dehydrogenase (S. K. Guha, unpublished) in the seminal plasma of RISUGinjected subjects.

It is well established that glycerophosphorylcholine originates mainly in the epididymis (Brown-Woodman et al., 1980). The physiological significance of glycerophosphorylcholine in epididymal secretion is not yet clear. Hartree and Mann (1960) suggested that glycerophosphorylcholine and phospholipids significantly affect respiration and motility of spermatozoa. There are controversial reports regarding the relationship between sperm motility and total seminal amounts of glycerophosphorylcholine (Arrata et al., 1978; Mieusset et al., 1988). The concentration of glycerophosphorylcholine and choline estimated in the present study was slightly higher than that reported after biochemical analysis by Mann and Mann (1981). The increased content of glycerophosphorylcholine may be attributed to the indistinguishibility of methyl resonances of glycerophosphorylcholine and phosphorylcholine. Even at a high field of $600 \mathrm{MHz}$, the glycerophosphorylcholine signal is nearly coincident with that of phosphorylcholine at 3.23 p.p.m. (Tomlins et al.,
1998). Tomlins et al. (1998) have also shown that unlike glycerophosphorylcholine, which is stable for several hours in human seminal fluid, phosphorylcholine is converted to choline with a half-life $\left(t_{1 / 2}\right)$ of $9 \mathrm{~min}$. There is also a partial overlapping of the $\mathrm{NCH}_{2}$ signal of spermine with the choline signal. These two factors are probably responsible for the higher concentration of choline recorded in the present study compared with standard biochemical data. The concentration of glycerophosphorylcholine was significantly lower in the seminal plasma of RISUG-injected men compared with control men. In RISUG-injected men, the partial obstruction of the vas deferens may slow down the flow of spermatozoa through the epididymis. The significant decrease in the sperm count of RISUG-injected subjects probably leads to a lowered epididymal activity. Lower seminal glycerophosphorylcholine concentrations have been reported in men with agenesis of the vas deferens (Calamera and Laveri, 1974) or after vasectomy (Frenkel et al., 1974; Naik et al., 1979; Giovenco et al., 1986). Jeyendran et al. (1989) reported a significant difference in glycerophosphorylcholine concentrations in fertile and infertile men, which indicates that glycerophosphorylcholine might influence the fertilizing ability of spermatozoa. ${ }^{1} \mathrm{H}$ NMR studies on seminal plasma from fertile and infertile men also showed that glycerophosphorylcholine, citrate and lactate concentrations in seminal plasma were significantly lower for patients with azoospermia compared with normal control groups (Hamamah et al., 1993). In the present study, significant differences were observed in citrate:lactate and glycerophosphorylcholine:choline ratios $(P<0.01)$ between control and RISUG-injected men. The estimated value of glycerophosphorylcholine:choline reported in the present study for RISUG-injected subjects is in excellent agreement with the ratio calculated by Hamamah et al. (1998) in men with obstructive azoospermia. However, the term 'partial obstructive azoospermia' would be more appropriate in the present study as ejaculates from RISUG-injected subjects do contain sperm debris (Guha, 1996).

Extensive overlapping of the resonances of metabolites and the nature of complexity of biofluid may act as limiting factors for accurate analysis of NMR spectra and, hence, the exact quantitation of metabolites. However, the aim of the present study was to study the relative differences between the two groups and, hence, the methodology used is appropriate.

In conclusion, the concentrations of prominent biochemical metabolites in seminal plasma, namely citrate, glucose, lactate, glycerophosphorylcholine and choline, were calculated using proton NMR spectroscopy and compared between RISUG-injected subjects and controls. Significantly lower concentrations of glucose, lactate, glycerophosphorylcholine and choline metabolites were observed in subjects injected with the contraceptive RISUG. The important outcome of the present study was the absence of a significant difference in the concentration of citrate in the seminal plasma of normal controls as well as in 
RISUG-injected subjects, which clearly rules out the possibility of a necrotic effect of RISUG on the prostate. Peak area ratios of various metabolites were also calculated and compared for control cases and RISUG-injected subjects. The glycerophosphorylcholine:choline ratio, in particular, indicated the occurrence of partial obstructive azoospermia in RISUG-injected subjects.

The study was supported by the Government of India, Ministry of Health and Family Welfare, and was conducted with the clinical trial approval of the Drugs Controller of India. The authors would like to thank the Chairman and members of the Ethics Committees for their understanding and for giving permission to carry out the experiments. The authors gratefully acknowledge the NMR facility provided by the All India Institute of Medical Sciences, New Delhi, for conducting the research work.

\section{References}

Arrata WSM, Burt T and Corder S (1978) The role of phosphate esters in male fertility Fertility and Sterility 30 329-333

Brown-Woodman PD, Marley PB, Morris S, Rodger JC and White IG (1980) Origin of glycerylphosphorylcholine, inositol, $\mathrm{N}$-acetylaminosugar, and prostaglandins in human seminal plasma and their effects on sperm metabolism Archives of Andrology 4 149-155

Calamera JC and Lavieri JC (1974) Glycerylphosphorylcholine in human seminal plasma of normal subjects and sterile patients Andrologia $\mathbf{6}$ $67-70$

Comhaire FH (1994) Male contraception: hormonal, mechanical and other Human Reproduction 9 586-590

Cooper TG, Yeung, CH, Nashan D, Jockenhovel F and Nieschlag E (1990) Improvement in the assessment of human epididymal function by the use of inhibitors in the assay of alpha-glucosidase in seminal plasma International Journal of Andrology 13 297-305

Cornel EB, Smits GAHI, Oosterhof GON, Karthaus HFM, Debruyne FMJ, Schalken JA and Heerschap A (1993) Characterization of human prostate cancer, benign prostatic hyperplasia and normal prostate by in vitro ${ }^{1} \mathrm{H}$ and ${ }^{31} \mathrm{P}$ magnetic resonance spectroscopy Journal of Urology $1502019-2024$

Costello LC and Franklin RB (1991) Concept of citrate production and secretion by prostate. I. Metabolic relationships Prostate 18 25-46

Diamandis EP, Arnett WP, Foussias G et al. (1999) Seminal plasma biochemical markers and their association with semen analysis findings Urology 53 596-603

Foxall PJ, Parkinson JA, Sadler IH, Lindon JC and Nicholson JK (1993 Analysis of biological fluids using $600 \mathrm{MHz}$ proton NMR spectroscopy: application of homonuclear two-dimensional J-resolved spectroscopy to urine and blood plasma for spectral simplification and assignment Journal of Pharmaceutical and Biomedical Analysis 11 21-31

Frenkel G, Peterson RN, Davis JE and Freund M (1974) Glycerylphosphorycholine and carnitine in normal human semen and in postvasectomy semen: differences in concentrations Fertility and Sterility $\mathbf{2 5}$ 84-87

Giovenco P, Calamera JC, Dondero F, Pacifici R and Vilar O (1986) Glycerylphosphorylcholine in selected forms of male infertility Andrologia 18 171-174

Guha SK (1996) Contraceptive for use by a male US Patent $\mathbf{5 4 8 8 0 7 0 5}$

Guha SK (1999) Non-invasive reversal of intraluminal vas deferens polymer injection induced azoospermia - technology Asian Journal of Andrology 1 131-134

Guha SK, Singh G, Anand S, Ansari S, Kumar S and Koul V (1993) Phase clinical trial of an injectable contraceptive for the male Contraception $48367-375$
Guha SK, Singh G, Ansari S, Kumar S, Srivastava A, Koul V, Das HC, Malhotra RL and Das SK (1997) Phase II of a clinical trial of a vas deferens injectable contraceptive for the male Contraception $\mathbf{5 6}$ 245-250

Hamamah S, Seguin F, Barthelemy C, Akoka S, Pape AL, Lansac J and Royere D (1993) ${ }^{1} \mathrm{H}$ nuclear magnetic resonance studies of human seminal plasma from fertile and infertile men Journal of Reproduction and Fertility $\mathbf{9 7}$ 51-55

Hamamah S, Seguin F, Bujan L, Barthelemy C, Mieusset R and Lansac J (1998) Quantification by magnetic resonance spectroscopy of metabolites in seminal plasma able to differentiate different forms of azoospermia Human Reproduction 13 132-135

Holmes E, Nicholls AW, Lindon JC et al. (2000) Chemometric models for toxicity classification based on NMR spectra of biofluids Chemical Research in Toxicology 13 471-478

Jeyendran RS, Van der Ven HH, Rosecrans R, Perez-Pelaez M, al-Hasani S and Zaneveld LJ (1989) Chemical constituents of human seminal plasma: relationship to fertility Andrologia 21 423-428

Liu M, Nicholson JK and Lindon JC (1996) High-resolution diffusion and relaxation edited one-and two-dimensional ${ }^{1} \mathrm{H}$ NMR spectroscopy of biological fluids Analytical Chemistry 68 3370-3376

Lynch MJ, Masters J, Pryor JP, Lindon JC, Spraul M, Foxall PJD and Nicholson JK (1994) Ultra high field NMR spectroscopic studies on human seminal fluid, seminal vesicle and prostatic secretions Journal of Pharmaceutical and Biomedical analysis 12 5-19

Mann T and Mann CL (1981) Biochemistry of seminal plasma and male accessory fluids; application to andrological problems. In Male Reproductive Function and Semen pp 272- 296 Eds T Mann and CL Mann. Springer-Verlag, Berlin Heidelberg

Mieusset R, Bujan L, Mansat A, Pontonnier F, Grandjean H and Chap H (1988) Glycerophosphocholine in seminal plasma of fertile and infertile men International Journal of Andrology 11 405-413

Naik VK, Pardanani DS, Joshi UM and Sheth AR (1979) Seminal plasma concentration of glycerylphosphorylcholine before and after vasectomy and vas reanastomosis Fertility and Sterility 32 685-686

Patel AB, Srivastava S, Phadke RS and Govil G (1999) Identification of lowmolecular weight compounds in goat epididymis using multinuclear nuclear magnetic resonance Analytical Biochemistry 206 205-215

Segalen J, De Certaines JD, Calve ML, Colleu D, Bansard JY and Rio M (1995) ${ }^{1} \mathrm{H}$ nuclear magnetic resonance on human seminal plasma attempts: use of automatic spectrum analysis Journal of Reproduction and Fertility 103 181-187

Sethi N, Srivastava RK and Singh RK (1990) Chronic toxicity of styrene maleic anhydride, a male contraceptive in rhesus monkeys (Macaca mulatta). Contraception 42 337-348

Sethi N, Srivastava RK, Nath D and Singh RK (1992) Teratological evaluation of an injectable male antifertility agent, styrene maleic anhydride International Journal of Fertility 37 183-187

Spraul M, Nicholson JK, Lynch MJ and Lindon JC (1994) Application of the one-dimensional TOCSY pulse sequence in $750 \mathrm{MHz}{ }^{1} \mathrm{H}-\mathrm{NMR}$ spectroscopy for assignment of endogenous metabolite resonances in biofluids Journal of Pharmaceutical and Biomedical Analysis 12 613-618

Tomlins AM, Foxall PJ, Lynch MJ, Parkinson J, Everett JR and Nicholson JK (1998) High resolution ${ }^{1} \mathrm{H}$ NMR spectroscopic studies on dynamic biochemical processes in incubated human seminal fluid samples Biochimica et Biophysica Acta 1379 367-380

World Health Organization (1999) Laboratory Manual for the Examination of Human Semen and Sperm Cervical Mucus Interaction pp 4-14 Ed. WHO. Cambridge University Press, Cambridge

Received 29 August 2000.

First decision 19 December 2000.

Revised manuscript received 18 April 2001.

Accepted 3 May 2001 